\title{
Spectroscopy Applied to Engineering Materials
}

\author{
Ming-Guo Ma, ${ }^{1}$ Wen Zeng, ${ }^{2}$ Shao-Wen Cao, ${ }^{3}$ Zhong-Chang Wang, ${ }^{4}$ and Jie-Fang Zhu ${ }^{5}$ \\ ${ }^{1}$ Beijing Key Laboratory of Lignocellulosic Chemistry, College of Materials Science and Technology, \\ Beijing Forestry University, Beijing 100083, China \\ ${ }^{2}$ College of Materials Science and Engineering, Chongqing University, Chongqing 400040, China \\ ${ }^{3}$ School of Materials Science and Engineering, Nanyang Technological University, Singapore 639798 \\ ${ }^{4}$ WPI Research Center, Advanced Institute for Materials Research, Tohoku University, Sendai 980-8577, Japan \\ ${ }^{5}$ Department of Materials Chemistry, Ångström Laboratory, Uppsala University, 75121 Uppsala, Sweden \\ Correspondence should be addressed to Ming-Guo Ma; mg_ma@bjfu.edu.cn
}

Received 15 December 2014; Accepted 15 December 2014

Copyright (C) 2015 Ming-Guo Ma et al. This is an open access article distributed under the Creative Commons Attribution License, which permits unrestricted use, distribution, and reproduction in any medium, provided the original work is properly cited.

For this special issue, we received 29 papers. Finally, 14 papers were selected for publication and more than $50 \%$ of the papers were rejected.

It is well known that engineering materials were always important tools in the technological development mainly due to their physical/chemical and mechanical properties in a wide range of applications such as machinery, vehicles, ships, construction, energy instrumentation, and aerospace. It is necessary to use spectroscopes analysis to study structure in engineering materials.

Within the published papers, various engineering materials were introduced such as lithium secondary batteries, hydrogen sensing materials, polymeric composites, highTC superconductor, cellulose film, carbon nanomaterial, and chitosan. In particular, most spectroscopes analysis methods were applied for the characterization of engineering materials including fluorescence spectroscopy, atomic force microscopy, diode laser-based photoacoustic spectroscopy, polarized micro-Raman spectroscopy, and Raman imaging.

Z. Xie and W. Guan reported three-dimensional fluorescence and infrared spectroscopy analysis of the leachate dissolved organic matter (DOM) of the Three Gorges in spring, summer, and autumn seasons, respectively. Experimental results indicated that three-dimensional fluorescence spectra of the landfill leachate varied with rubbish stacking time. They also reported that the longer the waste stacking time, the lower the protein in leachate concentration and the higher the fulvic-like acid concentration.

Y.-S. Kim and S.-K. Jeong investigated the electrochemical processes occurring at the surface of a highly ordered pyrolytic graphite electrode by in situ atomic force microscopy (AFM). Their results provided the direct and obvious evidence that solvent cointercalation reaction occurred at the initial stage of the surface film formation.

$\mathrm{X}$. Chen et al. used polarized micro-Raman spectroscopy as a powerful tool to map out the molecular orientation of a uniaxially oriented polypropylene-based composite material. Micro-Raman analysis at the surface region was found to demonstrate the surface orientation relaxation and provide an effective way to correlate the extent of relaxation and process conditions. J. L. González-Solís et al. also explored the chemical structure of $\mathrm{TlBa}_{2} \mathrm{Ca}_{2} \mathrm{Cu}_{3} \mathrm{O}_{9}$ high-TC superconductor films with Tl-1223 phase to monitor spectral map changes from normal state to superconducting state using the technique of Raman imaging, which provided the spatial distribution of the various molecular species within a sample. They reported that this is the first report of preliminary results evaluating the usefulness of Raman imaging in the determination of transition temperature of $\mathrm{TlBa}_{2} \mathrm{Ca}_{2} \mathrm{Cu}_{3} \mathrm{O}_{9}$ high-TC superconductor films with Tl-1223 phase.

This issue provided the recent development of advanced spectroscopic characterizations for engineering materials. 
Our expectation is that more attentions will be paid to the research of engineering materials.

\section{Acknowledgments}

The editors would like to take advantage of this opportunity to express their sincere thanks to the authors. They would like to thank them for submitting their best papers to this issue. Then, they also apologize for the long time of the review process. For example, it took 115 days to review E. Zumelzu et al's paper. Finally, the lead guest editor expresses his/her sincere gratitude to the editor team as it is not easy for them to assign the article to a reviewer.

Ming-Guo Ma

Wen Zeng

Shao-Wen Cao

Zhong-Chang Wang

Jie-Fang Zhu 

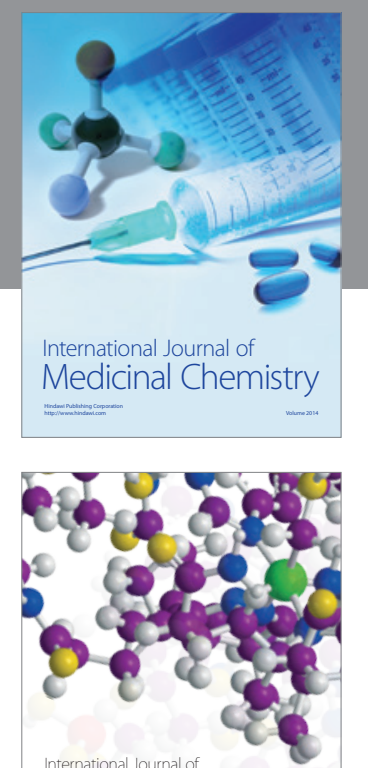

\section{Carbohydrate} Chemistry

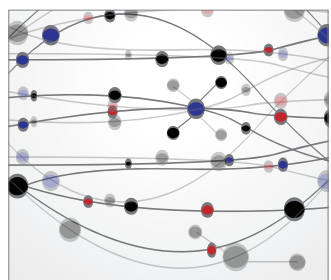

The Scientific World Journal
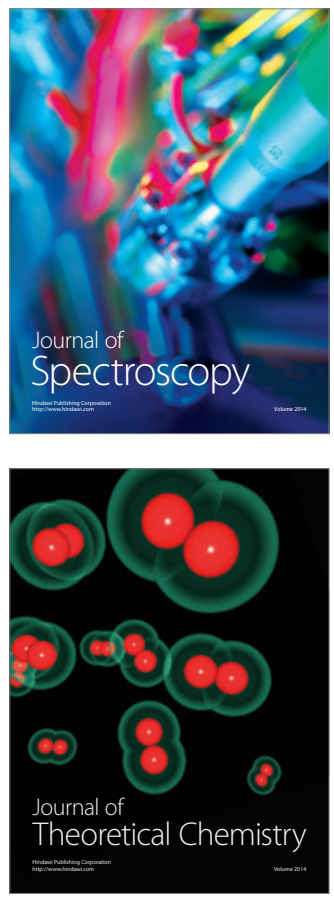
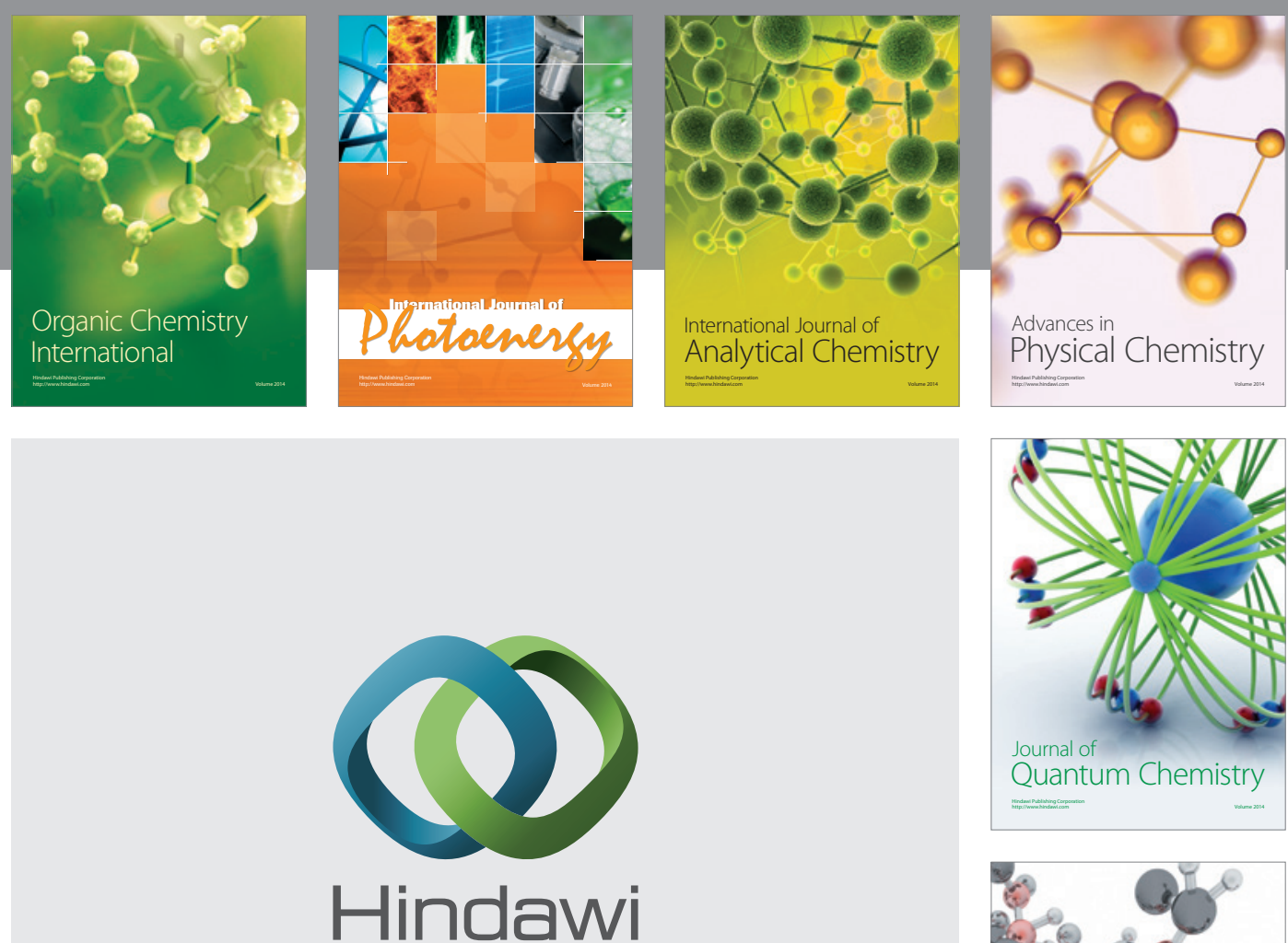

Submit your manuscripts at

http://www.hindawi.com

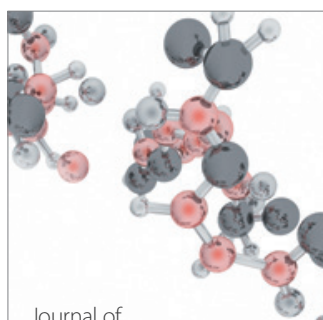

Analytical Methods

in Chemistry

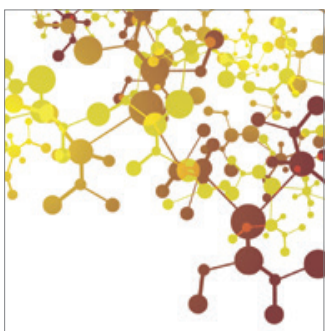

Journal of

Applied Chemistry

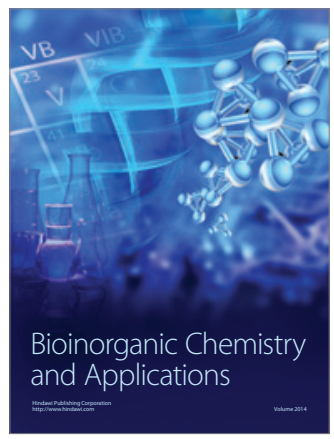

Inorganic Chemistry
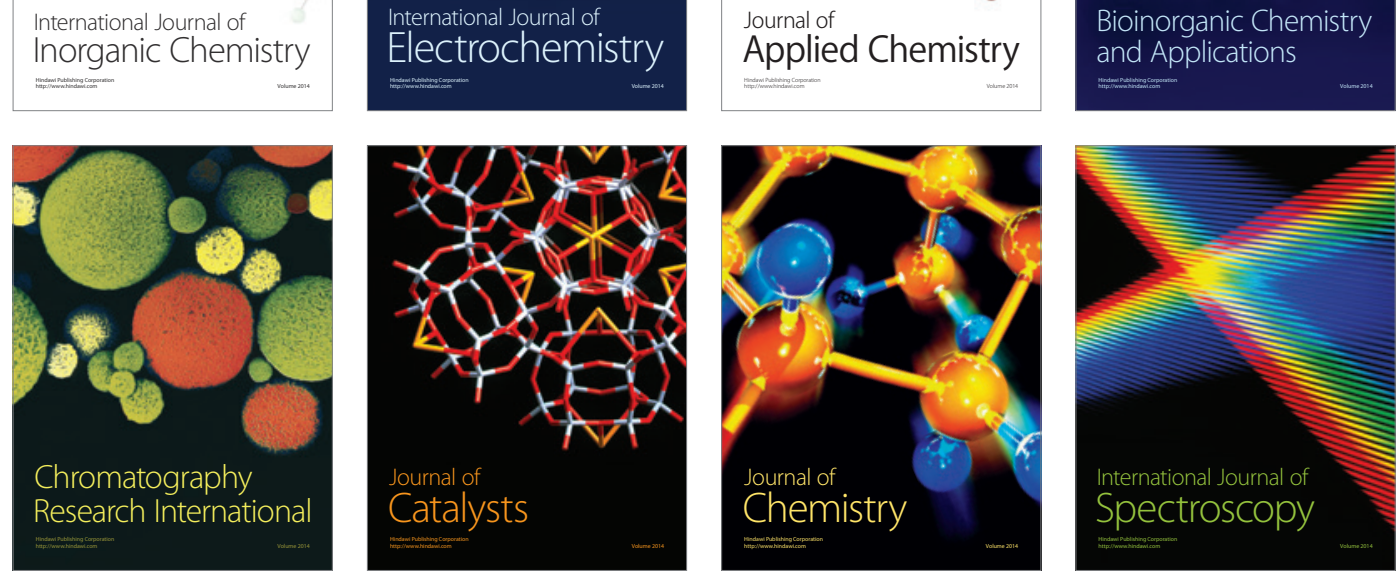